Arts

et Savoirs

\section{Arts et Savoirs}

$14 \mid 2020$

Styles de pensée, pensées du style. Écrire le vivant au XIXe siècle

\title{
Pierre Boitard : écrire le vivant dans le Musée des
}

familles

Pierre Boitard: writing about life in Musée des familles

\section{Eudes Huyghe}

\section{OpenEdition}

\section{Journals}

Édition électronique

URL : http://journals.openedition.org/aes/3335

DOI : $10.4000 /$ aes.3335

ISSN : 2258-093X

Éditeur

Laboratoire LISAA

Référence électronique

Eudes Huyghe, "Pierre Boitard : écrire le vivant dans le Musée des familles », Arts et Savoirs [En ligne],

14 | 2020, mis en ligne le 29 décembre 2020, consulté le 21 février 2021. URL : http://

journals.openedition.org/aes/3335; DOI : https://doi.org/10.4000/aes.3335

Ce document a été généré automatiquement le 21 février 2021.

Centre de recherche LISAA (Littératures SAvoirs et Arts) 


\title{
Pierre Boitard : écrire le vivant dans le Musée des familles
}

Pierre Boitard: writing about life in Musée des familles

\author{
Eudes Huyghe
}

1 "Il faut rendre la littérature populaire ", proclame Samuel Henry Berthoud dans une note aux lecteurs du Musée des familles ${ }^{1}$. Au début de l'année 1834, l'ancien imprimeur de Cambrai est conscient que la réussite des magasins populaires, dont fait partie le Musée des familles, dépend de leur capacité à s'adapter à leur public. Ces magasins d'instruction, dont la forme hybride, entre la revue littéraire et le journal hebdomadaire, est inédite dans le paysage éditorial français, visent un public familial composé à la fois de jeunes écoliers et d'adultes parvenus tardivement à la lecture, et proposent d'instruire et d'amuser un nouveau lectorat peu éclairé auquel peu d'éditeurs et d'auteurs se sont intéressés jusque-là. Cela suppose l'adaptation des procédés d'écriture au public visé, qu'il faut savoir séduire. Mais si cette "popularisation $»^{2}$ s'appuie d'abord sur les ressorts les plus évidents, la multiplication des illustrations, l'exclusion des thèmes sérieux comme la politique et l'économie générale, la réduction du prix d'abonnement, ces éléments ne sont pas suffisants pour donner naissance à une littérature "populaire »: inventer la littérature populaire suppose avant tout inventer une écriture.

2 Lorsque les magasins apparaissent, l'heure est au « pittoresque ». Si l'adjectif, issu de la peinture, rappelle que ces journaux sont illustrés, il constitue aussi une forme d'injonction dans le paysage éditorial des magasins : le Magasin pittoresque en a fait son nom autant qu'un argument de vente, tandis que de son côté, le Musée des familles multiplie les titres d'article qui en font la mention. La première année ne compte pas moins de onze articles sur «la France pittoresque», "l'Égypte pittoresque», les " costumes pittoresques", etc. Il faut donc peindre, Jules Janin s'y engage dans le premier numéro, appelant à dresser notamment le «portrait» de membres du règne animal ${ }^{3}$. Le terme de « portrait» ne va pourtant pas de soi : l'expression, employée par Jules Janin, est ici celle d'un homme de lettres, pas celle d'un naturaliste. Elle révèle une 
dimension aussi picturale qu'anthropomorphique des productions naturalistes du Musée des familles, héritières du style buffonien.

3 Au sein du Musée des familles, la part belle est faite à l'histoire naturelle dont la nature de science d'observation permet de satisfaire facilement le goût pour l'image de lecteurs friands de curiosités et d'exotisme. La rédaction des articles naturalistes est rapidement confiée à Pierre Boitard, un naturaliste autodidacte d'une trentaine d'années originaire de Mâcon ${ }^{4}$, qui devient rapidement le représentant emblématique de cette littérature naturaliste populaire qu'il fournit au magasin pendant presque quinze ans $s^{5}$. Son succès nous amène à nous interroger sur la manière dont Pierre Boitard est parvenu à répondre aux exigences éditoriales du Musée des familles: concilier instruction naturaliste et esthétique populaire, soit une littérature "amusante et utile " ${ }^{6}$. Mais nous verrons également que, chez Boitard, la popularisation de l'histoire naturelle ne s'est pas traduite par la mise à disposition à un public novice d'une science faite par les élites savantes mais, au contraire, par la promotion d'une science domestique, curieuse et praticable par le lecteur, pour laquelle Boitard a mobilisé de nombreux procédés stylistiques. Le style boitardien, qui n'est autre que le fruit d'un projet auctorial délibéré, engage une certaine conception de la science et se traduit dans un véritable «style de pensée ».

\section{Portraits du règne animal et contraintes éditoriales}

Lorsque Boitard entre au Musée des familles en septembre 1835, il devient le cinquième collaborateur à se charger de la rubrique d'histoire naturelle. Les trois premiers, Charles Nodier, Adélaïde Montgolfier et Alphonse Karr, l'avaient été de manière ponctuelle aux débuts du journal, tandis que le quatrième, à qui Boitard succède, Bory de Saint-Vincent, dont la réputation n'est pourtant plus à faire dans les sphères naturalistes, s'est parfois contenté de reprendre ou d'adapter pour l'occasion des notices ou des textes déjà publiés ${ }^{7}$. En signant l'ensemble des articles naturalistes de la troisième année et plus de la moitié de ceux des treize années suivantes, Boitard apporte une régularité qui faisait défaut lors des deux premières années du magasin. Contrairement à ses prédécesseurs, Boitard a le temps de développer son propre style.

Durant les huit premiers mois de sa collaboration avec le Musée des familles, Boitard se conforme au souhait des fondateurs du journal et dresse "pittoresquement ", espèce par espèce, des portraits d'animaux. Les articles courts, jamais de plus d'une page, prennent systématiquement place en fin de journal afin que les illustrations en pleinepage qui les accompagnent puissent constituer le verso des numéros mensuels. Les impressionnantes gravures - qu'il s'agisse d'ours, de crocodiles ou de chimpanzés deviennent des objets que le lecteur peut contempler en tant que tels, mais assurent aussi le rôle de vitrine pour des textes qu'il reste à découvrir8. Bien qu'on ne puisse savoir lequel, du texte ou de l'illustration, est réalisé en premier, les articles de Boitard sont, quoiqu'il en est, écrits pour faciliter la compréhension par le lecteur des gravures qui lui sont mis sous les yeux. Le style employé traduit dès lors l'importance que revêt le sens de l'observation, plus que le sens de la vue, au moment d'explorer les magasins. Ainsi, lorsque Boitard décrit le jaguar :

[Le jaguar] est d'un fauve vif, marqué sur les flancs de six rangées de taches noires formant l'anneau, avec un à quatre points noirs au milieu. On le distingue fort aisément de la panthère à son oreille dont le dessus est noir avec une large tache blanche lunulée, au milieu, tandis que la première a le dessus de l'oreille blanc avec 
une tache noire. Doit-on regarder comme espèces, ou simplement comme variétés, les jaguars dont les anneaux noirs des flancs sont ouverts et ceux dont les anneaux sont fermés ?

6 Il n'est pas question de simplement admirer l'animal mais d'être capable de le "distinguer» de la panthère. Boitard guide fermement le lecteur, depuis les taches noires des flancs jusqu'au-dessus des oreilles: le regard ne doit pas s'attarder sur les parties anatomiques qui n'ont pas d'utilité scientifique. La description est précise, rigoureuse, il n'y a pas "plusieurs » rangées mais très exactement «six », tandis que l'auteur décrit avec force détails la seule oreille du jaguar et la forme « lunulée » de sa tache blanche. Boitard met l'accent sur les formes et les couleurs, mais ce faisant il met en lumière les caractères diagnosiques ${ }^{10}$ du jaguar et profite en réalité des gravures ainsi créées pour initier le lecteur aux débats savants, où une seule description de quatre lignes devient prétexte à s'interroger sur le statut de l'espèce. On voit ici comment le caractère apparemment plaisant et bigarré de la description permet en réalité de conduire le lecteur à penser lui-même en naturaliste et à dépasser, presque sans s'en rendre compte, le pittoresque de la description pour en faire le fondement d'une véritable analyse scientifique.

7 À certains égards, la précision de ces gravures rappelle la tradition des planches anatomiques, tant Boitard prend la peine de minutieusement décrire ses sujets, couche par couche :

L'aï appartient à la classe des mammifères édentés. Il est de la grandeur d'un chat ;

il a des molaires cylindriques, des canines longues et pointues, une queue fort courte, les mamelles sur la poitrine, la tête arrondie et la face peu allongée. Ses yeux sont tristes, sans éclat; ses organes ne paraissent que de grossières ébauches et toute sa structure est informe ou ignoble. Son corps est couvert d'un poil long, entremêlé, ayant absolument la grosseur, la couleur et l'apparence d'herbe ou de mousse sèche. Ses pieds de devant sont embarrassés de trois gros ongles longs et crochus, ayant de l'analogie avec les sabots de plusieurs ruminans, mais recourbés sous la plante des pieds de manière à le gêner considérablement dans sa marche. Ses bras et ses avant-bras sont tellement plus longs que ses cuisses et ses jambes, qu'il est obligé de marcher sur ses coudes ; son bassin est beaucoup trop large pour lui permettre de joindre les genoux; enfin, ses pieds de derrière étant articulés obliquement sur la jambe, il ne peut les appuyer que par le côté externe. ${ }^{11}$

8 La dissection suit aléatoirement l'axe antéro-postérieur de l'animal, de la fourrure jusqu'aux os du squelette en passant par les organes, du haut du corps jusqu'aux pieds en remontant jusqu'aux bras. L'aï est épluché, décortiqué, scruté au cours d'une véritable vivisection, l'occasion pour l'anatomiste d'extraire sous les yeux du lecteur les particularités d'un animal qui tente encore de marcher. D'un point de vue méthodologique, l'opération pratiquée par Boitard est celle d'un véritable naturaliste, et son compte-rendu fait preuve d'un sens du détail indéniable. À ce titre, Boitard donne à son texte les apparences d'un texte savant, ce qui justifie sa présence au sein d'un magasin d'instruction. Mais le lecteur n'aura pas manqué de relever les qualificatifs que l'auteur attribue à l'aï : " tristes, sans éclat ", "grossières ébauches ", " informe ou ignoble", "ongles longs et crochus». Boitard sort du cadre rigoureux qu'exigerait une véritable description anatomique pour faire de l'animal une erreur de la nature, à partir de qualificatifs et de compléments du nom pleinement subjectifs. Une taille de chat, mais des pieds de ruminants, une démarche qui s'apparente à de la reptation, l'auteur va jusqu'à mettre en doute sa nature animale ( «la couleur et l'apparence d'herbe ou de mousse sèche ») : tout juste est-on étonné que les mamelles 
se trouvent bien sur la poitrine. Le contrat éditorial n'est pas rompu pour autant : en reprenant le vocable des gravures naturalistes, en guidant le regard du lecteur, en lui offrant l'expérience visuelle d'une authentique opération de dissection, Boitard assure la partie instructive qui lui incombe; en en déformant les codes, en interprétant l'œuvre de la nature, il procure la part d'amusement. En cela, il montre sa volonté de respecter les exigences éditoriales du Musée des familles.

\section{Familiariser le public : le style de la conversation}

Dans son œuvre, Boitard développe un lien avec le lecteur et favorise une lecture coopérative en mettant en place un cadre communicationnel, qui permet aussi bien l'interpellation du lecteur que la mise en scène de soi. Boitard met en place, dans ses portraits, une série d'éléments stylistiques qui visent à initier le dialogue, à la manière des discussions de salons de l'Ancien Régime. Ce processus est couronné par la mise en fiction des textes, qui permet de mettre en avant une véritable esthétique de la conversation. Les formules péremptoires («Il faut») sont abandonnées au profit de formules interrogatives («Doit-on? $»^{12}$ ), ce qui, au-delà d'intégrer les lecteurs à une réflexion savante commune et par là leur signifier que tout le monde appartient, ou tout du moins peut appartenir, à la communauté des naturalistes, marque l'intention de l'auteur de se positionner non pas en tant que professeur, mais en tant que savant, en tant que "celui qui sait", qui s'interroge et que l'on peut interroger. Les rares injonctions de l'auteur prennent la forme de consignes d'observation ${ }^{13}$ données à la première personne du pluriel, resserrant davantage le lien qui unit auteur et lecteurs. Par deux fois, Boitard se manifeste directement dans ses articles ${ }^{14}$, pour rapporter une anecdote ou pour témoigner de son expérience personnelle. Il faut cependant attendre Le tigre et l'hémione pour le voir s'adresser directement aux lecteurs ${ }^{15}:$ Boitard poursuit l'exploration du règne animal en s'adressant directement à son public (cette fonction phatique permet de resserrer les liens entre auteur et lecteur ${ }^{16}$ ), avant que, un mois plus tard ${ }^{17}$, son premier récit de fiction ne paraisse et, maintenant qu'auteur et lecteurs ont vu la familiarité gagner leurs échanges, de mettre en forme leurs premiers dialogues.

La fiction devient, pour Boitard, le moyen d'approfondir le dialogue scientifique. S'affranchissant du cadre imposé par l'exercice du portrait animalier, il apporte au récit une nouvelle dimension que l'esthétique de la conversation avait commencé à introduire. D'un point de vue stylistique, la connivence entre l'auteur et le lecteur prend alors de nouvelles formes, plus abouties. À ce titre, Paris avant les hommes constitue un texte-charnière. Ce récit autodiégétique met en scène Pierre Boitard luimême et Asmodée, le Diable Boiteux, au cours d'un voyage dans les époques passées. Ce diable, repris du roman éponyme d'Alain-René Lesage publié au début du siècle précédent, survole dans l'œuvre originale les toits de Madrid pour révéler les secrets des intérieurs domestiques. Ce choix est donc significatif: Boitard fait du diable le guide chargé de dévoiler au narrateur, et donc au lecteur, les mystères du monde passé. Ce génie, que le lecteur ne peut s'empêcher de trouver attachant ${ }^{18}$, avec lequel l'auteurnarrateur entretient un rapport ambigu, tantôt admiratif ${ }^{19}$, tantôt craintif ${ }^{20}$, tantôt moqueur ${ }^{21}$, ne trompe personne et la presque homonymie Boiteux/Boitard est là pour nous le rappeler: Boitard utilise le personnage du Diable Boiteux pour construire sa figura d'auteur. En se faisant l'acolyte d'un guide "sulfureux », il révèle la manière dont 
il se perçoit dans l'univers des naturalistes, en même temps que, peut-être, sa conception du rôle que doivent jouer les sciences du vivant comme révélateurs de vérités cachées et sans doute dérangeantes. À cinq reprises, le possessif «mon » est associé au génie : «Mon génie me dit que... », ou « Pourquoi, dis-je à mon génie, [...] », laissant apparaître en filigrane, par l'usage polyphonique d'une certaine forme de prosopopée, un dialogue intérieur de Boitard avec lui-même, dont l'effet est renforcé par la révélation finale que le voyage temporel n'est qu'un simple rêve.

11 La relation qui unit l'auteur à ses lecteurs se fait par ailleurs plus étroite. Le lecteur découvre Pierre Boitard chez lui, dans l'intimité de son cabinet, dont Boitard communique l'adresse ${ }^{22}$. L'auteur faisant référence à un thermomètre "acheté chez l'habile M. Delamarre » (p. 260) et à un "galop de M. Musard» (p. 262) comme si les lecteurs passaient régulièrement dans la boutique du premier et dansaient sur les musiques du second à chaque bal. De la même manière, Boitard destine ses contes à ses lecteurs jusqu'à prendre en considération leur sensibilité au moment d'entamer un nouveau voyage avec le diable boiteux. Ainsi dans la première partie de Voyage dans le Soleil :

Ce guide c'était le diable boiteux, qui déjà m'avait montré Paris avant les hommes. Ce que je l'entendais me dire, ou si vous voulez ce que je croyais l'entendre me dire était quelquefois si extraordinaire, si bizarre, que vraiment je n'oserai vous le répéter comme venant de moi. Ainsi donc, pour vous raconter ce que j'ai vu, il faut absolument que je lui laisse le rôle dont il s'était chargé, et, soit que vous le preniez pour une fiction inventée tout exprès pour me mettre à l'aise, soit que vous le regardiez comme l'enfant d'une imagination un peu délirante, il faut que vous l'acceptiez comme je l'acceptai moi-même. Du reste, si je vous garantis la vérité de tout ce qui sortira de ma bouche, il s'en faut de beaucoup que je prenne le même engagement pour ce qui sortira de la sienne, car on sait que les démons, ainsi que tous les enfans de l'imagination, sont naturellement enclins aux utopies. ${ }^{23}$

12 La référence à Paris, autant que les expressions «extraordinaire » et «bizarre » traduisent une stratégie publicitaire de la part de l'auteur, qui attise la curiosité des lecteurs en leur faisant miroiter d'autres aventures fantastiques. L'écriture de la relation entre Boitard et le Boiteux entre dans une dimension ludique poussée à l'extrême: l'auteur se dissocie formellement du génie, et lui confère une existence propre et indépendante, maintenant l'ambiguïté du personnage sur un registre presque pathologique. Mais ce qui nous intéresse ici est la précaution avec laquelle Boitard place son lectorat dans de bonnes conditions de lecture: «Si vous voulez...», «je n'oserai vous le répéter... », «je vous garantis la vérité... ». Les termes du contrat de lecture sont exposés de manière explicite ( il faut que vous l'acceptiez comme je l'acceptai moi-même »), comme pour sous-entendre que les règles du discours ne sont pas les règles d'un texte naturaliste traditionnel. Si Boitard n'en est pas au point de recruter des adeptes dans les rangs des lecteurs du Musée des familles, la considération avec laquelle il s'adresse à eux traduit néanmoins la recherche d'une certaine forme d'engagement de leur part, une façon de leur signifier que le discours savant que l'auteur leur soumet est bien distinct du discours académique ordinaire. 


\section{Déshabiller les élites : caricature du style académique et promotion du style domestique} Boitard, trait qu'il ne cherche pas à dissimuler par son style d'ecriture, mais contraire à mettre en évidence. Cet anti-académisme se traduit, chez lui, de trois manières distinctes. comme ceux du narrateur («Vos savants!») puisque le diable a l'habitude de tutoyer ce dernier. Lorsque le diable est en colère, le vouvoiement est de mise, comme le confirment d'autres formules ("vos barbouilleurs de Paris », p. 262 ; «vos géologues », p. 266). Tout, finalement, revient à une caricature des sphères savantes. Les traits sont exagérés : que Boitard évoque la moralité des hommes de science qui « ne [parlent] que par sentences $»^{25}$, leur aspect physique de vieillards malades ${ }^{26}$, ou qu'il réduise les savants, là encore par métonymie, à de simples «nomenclateurs " ${ }^{27}$ et leur science à du " savantisme $~^{28}$ - dont la présence du suffixe "-isme » apparente ce dernier à une doctrine, voire à pratique douteuse ${ }^{29}$ comme le charlatanisme -, chaque texte devient une opportunité de ridiculiser les naturalistes et leur science. Aussi Boitard tourne-t-il ces derniers en dérision, hyperbolisant le temps consacré à «disséquer l'œil d'une mouche ", ironisant sur leur capacité à "presque [trouver] le moyen de distinguer un lapin d'avec un chou » ou sur l'utilité qu'a leur savoir sur le «bonheur des hommes ». ${ }^{30}$ La critique boitardienne des élites est générale, l'usage de la caricature à cet effet ne l'est pas moins.

D'autre part, le discours scientifique de Boitard prend le contre-pied des discours académiques classiques. Ce contre-pied s'observe aussi bien dans la présence d'anecdotes que dans celle d'une certaine forme de bouffonnerie, ou dans celles d'idées scientifiques rejetées dans le milieu académique. Dix ans avant de devenir collaborateur au Musée des familles, Boitard déplorait déjà que les traités naturalistes ne permettent pas d'y rapporter quelques anecdotes ${ }^{31}$, une contrainte qu'il s'empresse d'outrepasser dès ses premiers articles pour le journal. Ces anecdotes constituent alors des preuves d'autant plus grandes que l'histoire est particulière, ce qui ne manque pas d'être souligné par antithèse: «tout le monde» connaît l'histoire du "petit ramoneur $\|^{32}$. Les caractères spécifiques des animaux décrits sont mis en évidence par des énumérations proposées sur un rythme ternaire ${ }^{33}$, et le vocabulaire pathologique prend le pas sur la rigueur scientifique des descriptions. Histoires et légendes populaires sont ainsi rapportées, conférant aux articles naturalistes de Boitard une note plus légère, propre à amuser le lecteur ou à l'émouvoir ${ }^{34}$, loin du ton exigé pour un article traditionnel.

17 Enfin, l'anti-académisme de Pierre Boitard se traduit par la promotion d'une science alternative, construite en opposition, voire en concurrence, avec la science 
académique. Cette science, que l'on peut qualifier de science "domestique", se doit d'être praticable par tout le monde, et surtout par le lecteur. Chaque exploration de son environnement peut ainsi devenir l'occasion de mener une étude naturaliste, qu'il s'agisse de faire le tour de son jardin ${ }^{35}$ ou de se promener sur les chemins champêtres ou urbains ${ }^{36}$. Cette incitation à « promener [ses] rêverie[s] » fait son apparition dans la première partie des " Réalités fantastiques » (vol. III, 1836) dans laquelle Boitard, usant de l'anaphore ( «Je veux...») et filant la métaphore du livre ${ }^{37}$, annonce son intention d'aider le lecteur à " déchiffrer » la nature et de le « convaincre » de l'intérêt de cette science domestique. C'est bien Boitard qui prend la parole: le «je» est l'unique pronom des premiers paragraphes, avant que n'apparaisse le «vous» par lequel l'auteur s'adresse directement à ses lecteurs. Les « bois de Boulogne, de Meudon ou de Saint-Cloud» sont autant de lieux où la flânerie doit permettre de "saisir [le merveilleux] à chaque pas » et où Boitard se propose de servir de guide. La science "domestique » se caractérise par cette proximité avec le lecteur, marquée par l'usage de l'impératif à la première personne du pluriel ${ }^{38}$. Les espèces exotiques, telles que l'oursin ou l'ornithorynque, sont systématiquement comparées à des éléments communs et familiers ${ }^{39}$, ancrant encore un peu plus les descriptions de Boitard dans l'environnement direct de ses lecteurs.

\section{Conclusion}

18 À partir de 1835, Pierre Boitard est à l'origine d'une nouvelle forme d'écriture naturaliste au sein du Musée des familles, caractéristique de ce que l'on peut considérer comme une littérature naturaliste populaire. Afin de répondre aux attentes du public visé et aux exigences éditoriales, Boitard entame une démarche de popularisation de l'histoire naturelle. Ce dessein scientifique s'est avant tout traduit par la mise en scène d'une conversation menée avec les lecteurs, sans crainte du ridicule pour l'auteur qui se met lui-même en scène. Par une série de portraits animaliers puis d'un ensemble de contes scientifiques qui lui permettent de mettre en fiction l'histoire naturelle, Boitard abandonne les codes et les raisonnements traditionnels pour une science nonacadémique fondée sur une démarche plutôt que sur l'apprentissage des nomenclatures. Parallèlement, il construit sa figura d'auteur et son projet, tous deux représentatifs d'un style de pensée qui marque sa volonté de s'inscrire comme un homme de science à part entière, à défaut de pouvoir devenir un acteur du monde savant dont il rejette les codes.

Critique abondante et générale des institutions savantes et de leurs membres, promotion d'une science domestique qui se caractérise par une priorité donnée à l'observation, à la pratique et à l'émerveillement, l'entreprise de popularisation mène à la proposition et à la promotion d'une science à la fois alternative et d'opposition, à des lecteurs dont les pages du Musée des familles peuvent devenir la première rencontre avec l'histoire naturelle. Ce projet devient en fin de compte représentatif de la littérature naturaliste proposée par le Musée périodique à ses lecteurs. La longévité de Boitard au sein du magasin témoigne de la confiance que les ses différents rédacteurs lui ont accordée, et dans une certaine mesure du succès qu'il a rencontré auprès du public. Le libraire-éditeur Passard compilera, agrémentera, et éditera ces contes savants à la mort de l'auteur en 1859 , démontrant que vingt ans après leur parution, ces derniers pouvaient encore avoir des lecteurs. 
20 a question reste de savoir si le style boitardien est propre au magasin, ou représentatif d'une option de défiance et d'irrévérence vis-à-vis des sphères savantes, à l'image de son collaborateur Alphonse Karr. À ce titre, l'étude des textes de Boitard pour le Magasin universel, et en particulier celle de L'homme fossile ${ }^{40}$, réécriture de Paris avant les hommes parue en 1838, apporterait des éléments de réponse.

\section{NOTES}

1. Samuel Henry Berthoud, « Aux lecteurs du Musée des familles », Musée des familles, I, avril 1834, p. 201.

2. Nous privilégions cette expression par rapport à celle de "vulgarisation », que nous préférons réserver aux textes issus des journaux spécialisés de la seconde moitié du XIX ${ }^{\mathrm{e}}$ siècle et qui témoigne d'une autre approche de la littérature scientifique.

3. "Nous ne négligerons pas plus un beau tigre qu'un beau cheval; et, après avoir donné le portrait de quelque grand homme du jour, nous ne croirons pas déroger en donnant le portrait d'un lion magnifique ou d'un beau poisson. » (Janin Jules, "Les magasins anglais », Musée des familles, I, octobre 1833, p. 6)

4. Pierre Boitard (1789-1859) arrive à Paris vraisemblablement à la fin de l'année 1820. D'abord rédacteur pour le Journal des débats, il est remarqué par le libraire Louis-Eustache Audot qui le recrute pour contribuer à la rédaction de la collection de La Botanique des dames dès 1821. Boitard signe ainsi huit traités et devient collaborateur au sein de quatre revues d'agronomie et de botanique. Il rejoint le libraire Nicolas-Edme Roret en 1825 qu'il ne quittera que vingt-cinq ans et une trentaine d'ouvrages plus tard. Boitard est nommé Chevalier de la Légion d'Honneur en juillet 1835, quelques semaines avant d'entrer au service du Musée des familles.

5. Peu d'auteurs se sont intéressés à l'œuvre de Pierre Boitard (plus rares encore sont ceux à avoir abordé dans leurs travaux la question du style). Pour autant, nous renvoyons le lecteur aux travaux de référence suivants: Claude Blanckaert, «Devilish Words: Pierre Boitard, "maître Georges" and the Advance of Nature ", Biological Time, Historical Time. Transfers and Transformations in $19^{\text {th }}$ Century Literature, Niklas Bender et Gisèle Séginger (dir.), Leyde/Boston, Brill/Rodopi, 2019, p. 45-60; Azélie Fayolle, «La vulgarisation excentrique pour tous : les leçons d'histoire naturelle de Pierre Boitard dans le Musée des familles ", dans Littérature française et savoirs biologiques au XIX siècle, G. Séginger, T. Klinkert (dir.), Berlin, De Gruyter, 2019, p. 213-231.

6. Samuel Henry Berthoud, «Aux lecteurs du Musée des familles », Musée des familles, I, avril 1834, p. 202.

7. La lecture de l'article «Le Banyan » dans le premier volume du Musée des familles, juillet 1834 (p. 288), et celle du «Banyan tree» dans The Oriental annual, or Scenes in India (Londres, Edward Bull, 1834) de Daniell et Caunter (p. 185) ne laissent place à aucun doute à ce sujet.

8. Il n'est pas à exclure que ces illustrations figuraient dans les vitrines des libraires pour assurer la promotion du Musée des familles. Un tel procédé publicitaire ne serait pas inconcevable au vu de la véritable campagne publicitaire menée par le Musée des familles au moment de son lancement, et qui constituait une pratique toute nouvelle pour l'époque.

9. Pierre Boitard, «Le jaguar », Musée des familles, III, décembre 1835, p. 94.

10. Les caractères « diagnosiques » sont les caractères propres d'une espèce, et qui par extension permettent de la distinguer d'une autre. 
11. Pierre Boitard, «L'aï et le condor », Musée des familles, III, février 1836, p. 158.

12. Pierre Boitard, « Le jaguar », Musée des familles, III, décembre 1835, p. 94.

13. «Mais détournons des yeux attristés de cet exemple de ce que la fatalité peut accumuler de maux sur un être innocent, et portons-les sur son ennemi le plus dangereux après l'homme, sur le condor ». (Pierre Boitard, «L'aï et le condor », Musée des familles, III, février 1836, p. 159.)

14. «Je ne puis résister à l'envie de vous rapporter un de ces contes; le voici : [...]»; puis «Enfin j'ai vu quatre ou cinq tigres vivants dans le cours de ma vie; tous étaient parfaitement apprivoisés et ne montraient pas un caractère plus féroce que le lion » (Pierre Boitard, «Le tigre et l'hémione ", Musée des familles, III, mai 1836, p. 255.)

15. «Or, vous remarquerez qu'il s'agit bien ici du véritable tigre; car, à la description que Pline nous a laissée de cet animal [...] » (Pierre Boitard, «Le tigre et l'hémione », Musée des familles, III, mai 1836, p. 255.)

16. Cette manière de s'adresser directement aux lecteurs fait partie de la culture du Musée, qui insère régulièrement des « Notes aux lecteurs" au cours de ses numéros, généralement tous les six mois.

17. Clôturant le numéro de mai 1836 avec Le tigre et l'hémione et inaugurant celui de juin 1836 avec Paris avant les hommes, cette proximité chronologique est d'autant plus significative sur le papier qu'aucun texte ne vient s'insérer entre les deux.

18. " $\mathrm{Au}$ fond, je suis un bon diable... » (Pierre Boitard, "Paris avant les hommes ", Musée des familles, III, juin 1836, p. 258)

19. "Voilà un diable qui doit être véritablement savant pensai-je, car il ne se pique pas de tout savoir », (Pierre Boitard, « Paris avant les hommes », Musée des familles, III, juin 1836, p. 258)

20. «Par prudence je coupai court à la dissertation, car je vis que la figure de mon démon commençait à se barioler de rouge et de violet... », (Pierre Boitard, " Paris avant les hommes ", Musée des familles, III, juin 1836, p. 266)

21. «...de rouge et de violet, comme une truite saumonée du lac de Genève.» (Pierre Boitard, «Paris avant les hommes », Musée des familles, III, juin 1836, p. 266)

22. " - Ton cabinet est dans le faubourg Saint-Germain, me dit-il ? - Oui. - Rue du CimetièreSaint-André-des-Arts ? - Oui. - Eh bien ! lève le nez en l'air et regarde. » (Pierre Boitard, «Paris avant les hommes ", Musée des familles, III, juin 1836, p. 258)

23. Pierre Boitard, « Voyage dans le Soleil », Musée des familles, VI, décembre 1838, p. 66.

24. Pierre Boitard, « Paris avant les hommes », Musée des familles, III, juin 1836, p. 260.

25. «[Ce savant] que tout le monde admirait et consultait, qui tranchait despotiquement sur toutes les questions, qui ne parlait que par sentences, [...] qui marchait la tête si haute qu'il eût, avec les pieds, écrasé les petits enfants sans les voir » (Pierre Boitard, "Fatima ", Musée des familles, IV, juillet 1837, p. 306)

26. "Alors mes cheveux devinrent blancs, mes membres raides, mes pieds goutteux, et mon caractère triste et irritable. J'étais malheureux et ennuyé. » (Pierre Boitard, «Fatima », Musée des familles, IV, juillet 1837, p. 307). Le personnage du Sage, ancien savant repenti, raconte son expérience d'homme de sciences en France.

27. «Le nomenclateur Temminck, qui, ainsi que tous les autres nomenclateurs, prend souvent la méthode arbitraire pour la marche de la nature, a aussi établi trois sections dans le genre parus [...]. Nous terminerons cet article en regrettant que la science, surtout en ornithologie, soit étouffée de plus en plus sous une synonymie effrayante, et cela est le malheureux résultat de l'amour propre des collectionneurs qui, pour se donner de l'importance, ne pouvant enrichir l'histoire naturelle par des faits, croient l'enrichir par des mots. M. Temminck est moins qu'un autre à l'abri de ce reproche, et cependant $\mathrm{M}$. Temminck était placé pour devenir naturaliste, et le disputer, peut-être, à nos savants professeurs du muséum d'Histoire naturelle. (Pierre Boitard, «La mésange à moustache », Le Magasin universel, VI, Paris, février 1839, p. 150.) 
28. «Déjà, me disposant à vous conduire par la main à travers les merveilles de la nature, j'ai jeté là le masque du savantisme, la pédanterie. » (Pierre Boitard, "La mésange à moustache ", Le Magasin universel, VI, Paris, février 1839, p. 150.)

29. "Savantisme » apparaît dans la deuxième édition du Dictionnaire de mots nouveaux, de JeanBaptiste Richard de Radonvilliers, édité chez Léautey, 1845

30. "Je sais que les oiseaux ont des plumes et pas de mamelles, et que les animaux qui ont des mamelles n'ont pas de plumes. J'ai passé trois ans de ma vie à disséquer l'œil d'une mouche, l'aiguillon d'une guêpe et trois pattes d'araignée ; j'ai presque trouvé le moyen de distinguer un lapin d'avec un chou, ou, si vous aimez mieux, un animal d'avec une plante, ce que n'avaient pu faire les savants avant moi. Je sais que les chenilles ont trois cents paires de nerfs, que les hannetons ont quatre ailes, que les mouches n'en ont que deux, découverte précieuse sur laquelle est établie la classification entomologique. Je sais que les serpents n'ont pas de pattes, les grenouilles pas de queue, et mille autres choses d'une aussi haute utilité pour le bonheur des hommes. » (Pierre Boitard, « Fatima », Musée des familles, IV, juillet 1837, p. 307).

31. «Dans quelques pays montagneux de la France, on s'amuse aussi à leur donner la chasse, et l'on emploie pour les prendre plusieurs procédés que nous ne décrivons pas, parce qu'ils n'entrent point dans le cadre de cet ouvrage. » (Pierre Boitard, Les Pigeons de volière et de colombier, Paris, Audot, 1824, p. 153.)

32. Pierre Boitard, «Les ours ", Musée des familles, III, octobre 1835, p. 31.

33. "Sa violence, ses accès, sa fureur... " (Pierre, Boitard, "Les ours ", Musée des familles, III, octobre 1835, p. 31.) ; « [Les chimpanzés] savent très bien se réunir pour attaquer, chasser, et même tuer... " (Pierre Boitard, "Le Chimpansé », Musée des familles, III, janvier 1836, p. 127.); "L'aï est entouré de nombreux ennemis, et pour toute arme à leur opposer il n'a que sa résignation, son impuissance et sa timidité » (Pierre Boitard, "L'aï et le condor ", Musée des familles, III, février 1836, p. 159.)

34. "Masco était un ours renfermé dans une cage du palais; sa violence, ses accès, sa fureur, lorsqu'on l'irritait, lui avaient valu dans le pays une grande réputation de férocité, passée en proverbe dans le pays ; on disait : mauvais comme Masco. Il se fit qu'un pauvre petit ramoneur de cheminée, ne sachant où dormir par une froide nuit d'hyver, s'avisa, dans un moment de désespoir, d'entrer dans la cage de Masco, en passant entre deux barreaux, et de s'y blottir sans bruit. Masco s'aperçut bientôt de la présence de son hôte ; mais au lieu de lui faire mal, il le réchauffa, le prit en amitié, et le reçut chaque nuit. L'enfant vint à mourir de la petite vérole ; dès ce moment l'ours refusa toute nourriture, et mourut. " (Pierre Boitard, «Les ours », Musée des familles, III, octobre 1835, p. 31.)

35. Boitard, Pierre, «La Très-véridique histoire des dix-neuf infortunes de Jannot le harponneur ", Musée des familles, XIII, février 1846.

36. «Moi aussi j'aime le merveilleux; mais trop paresseux pour courir après, je veux le trouver sous ma main quand je promène mes rêveries sur les bords d'un ruisseau ou sous l'ombrage silencieux des forêts » (Pierre, Boitard, « Réalités fantastiques », Musée des familles, III, août 1836, p. 326).

37. «Là, cent peuples divers m'ouvrent leurs singulières archives, et il ne manque dans ces archives ni fantastique ni merveilleux pour ceux qui savent y lire. Je veux vous en faire déchiffrer quelques pages avec moi, car mon intention est de vous convaincre. » (Pierre, Boitard, « Réalités fantastiques ", Musée des familles, III, août 1836, p. 326).

38. «Greffons-le autrement » (p. 187) ; «Prenons un de nos polypes» (p. 187); «Exposons-les d'abord un instant » (p. 190), etc. (Pierre, Boitard, « Réalités fantastiques », Musée des familles, IV, mars 1837)

39. «L'oursin commun a la forme et la grosseur d'une pomme» (IV, p. 190); "le crâne de l'ornithorynque se prolonge antérieurement en un véritable bac de canard» (IV, p. 182) ; etc.

40. Pierre Boitard, « L'homme fossile », Magasin universel, V, avril 1838, p. 209-240. 


\section{RÉSUMÉS}

Par leur forme inédite et en ciblant un lectorat populaire peu instruit, les magasins populaires, apparus au début des années 1830, ont incité leurs auteurs à inventer une nouvelle littérature. Au sein du Musée des familles, Pierre Boitard a ainsi été chargé de la rédaction de leçons naturalistes. Souhaitant promouvoir une science domestique, mise en concurrence avec la science des institutions savantes qu'il abhorrait, son style, caractérisé par la mise en place d'une esthétique de la conversation, d'une proximité avec le lecteur et d'une critique systématique des élites, est devenu aussi bien un outil mis au service de son projet scientifique qu'un témoin d'un véritable style de pensée.

Through their novel form and by targeting a poorly educated working-class readers hip, popular magazines, first published in the early 1830s, incited their authors to invent a new form of literature. Within the Musée des familles, Pierre Boitard was thus tasked with writing naturalistic lessons. As he wished to promote a form of domestic science, as opposed to the science of scholarly institutions he abhorred, he developed a style characterized by conversation esthetics, proximity with the reader, and a systematic criticism of the elites. His style became as much a tool serving his scientific project as it was the testimony to his very style of thinking.

\section{INDEX}

Mots-clés : Boitard (Pierre), littérature populaire, style de pensée, leçons naturalistes, science domestique

Keywords : Boitard (Pierre), popular literature, style of thinking, naturalistic lessons, domestic science

\section{AUTEUR \\ EUDES HUYGHE}

EA 4195 - TELEM, Université Bordeaux Montaigne 